\title{
The Importance of Three Dimensional Electroanatomical Mapping in Radiofrequency Ablation of Scar Related Atrial Flutter
}

\author{
Szilamér Korodi, András Mester², István Kovács ${ }^{1}$ \\ ${ }^{1}$ Clinic of Cardiology, University of Medicine and Pharmacy, Tîrgu Mureș, Romania \\ 2 Center of Advanced Research in Multimodality Cardiac Imaging, Cardio Med Medical Center, Tîrgu Mureș, Romania
}

\section{CORRESPONDENCE}

\section{András Mester}

76, 22 Decembrie 1989 St 540124

Tîrgu Mureș, Romania

Tel: +40 265217333

Email: andras.mester@yahoo.com

\section{ARTICLE HISTORY}

Received: 10 May, 2016

Accepted: 16 May, 2016
Szilamér Korodi • 38 Gheorghe Marinescu St, 540139 Tîrgu Mureș, Romania, Tel: +40 265215551 Email: szilamerkorodi@yahoo.com

István Kovács • 38 Gheorghe Marinescu St, 540139 Tîrgu Mureș, Romania, Tel: +40 265215551

Email: kov_istvan@yahoo.com
A 72 year-old woman presented to our hospital with recurrent palpitations associated with worsening shortness of breath. She had a history of severe mitral valve stenosis requiring mitral valve commissurotomy. Electrocardiogram showed flutter waves with 138 beats per minute (bpm) (Panel A). After obtaining written consent, an electrophysiological study was performed. After right femoral vein puncture, we introduced an ablation catheter into the high right atrium, a duodecapolar catheter (HALO) into the right atrium, and a steerable decapolar catheter into the coronary sinus. Basic electrophysiological recordings showed an atypical atrial flutter with a 164 msec right atrial activation time and a $234 \mathrm{msec}$ supraventricular tachycardia cycle length (STCL) (Panel B). Entrainment pacing was performed from the antero-lateral wall of the right atrium. The difference of the post-pacing interval (PPI) and the STCL after the entrainment pacing shows the closeness of the entrainment catheter to the reentry circuit, and the PPI-STCL at the antero-lateral wall of the right atrium was $<10 \mathrm{msec}$. (Panel C). We performed three-dimensional electroanatomical mapping (Panel D) with Ensite NavX system. The activation (Panel E) and voltage mapping (Panel F) confirmed that the reentry circuit was located on the antero-lateral wall of the right atrium, above the inferior vena cava. We performed radiofrequency ablation with an irrigated, contact force sensing ablation catheter (TactiCath Quartz 75, St Jude Medical) on the anterior side of the scar (Panel G). The applied radiofrequency current power was $50 \mathrm{~W}$, the temperature limit was $55{ }^{\circ} \mathrm{C}$, and the applied maximum contact force was $17 \mathrm{~g}$. The reentry circuit was successfully ablated (Panel $\mathrm{H}$ and Panel I). At the 1 month follow-up the patient was free of any symptoms or arrhythmia.

The ablation of atypical flutter has a lower success rate than the ablation of the typical cavotricuspid isthmus-dependent atrial flutter. ${ }^{1-3}$ The use of 


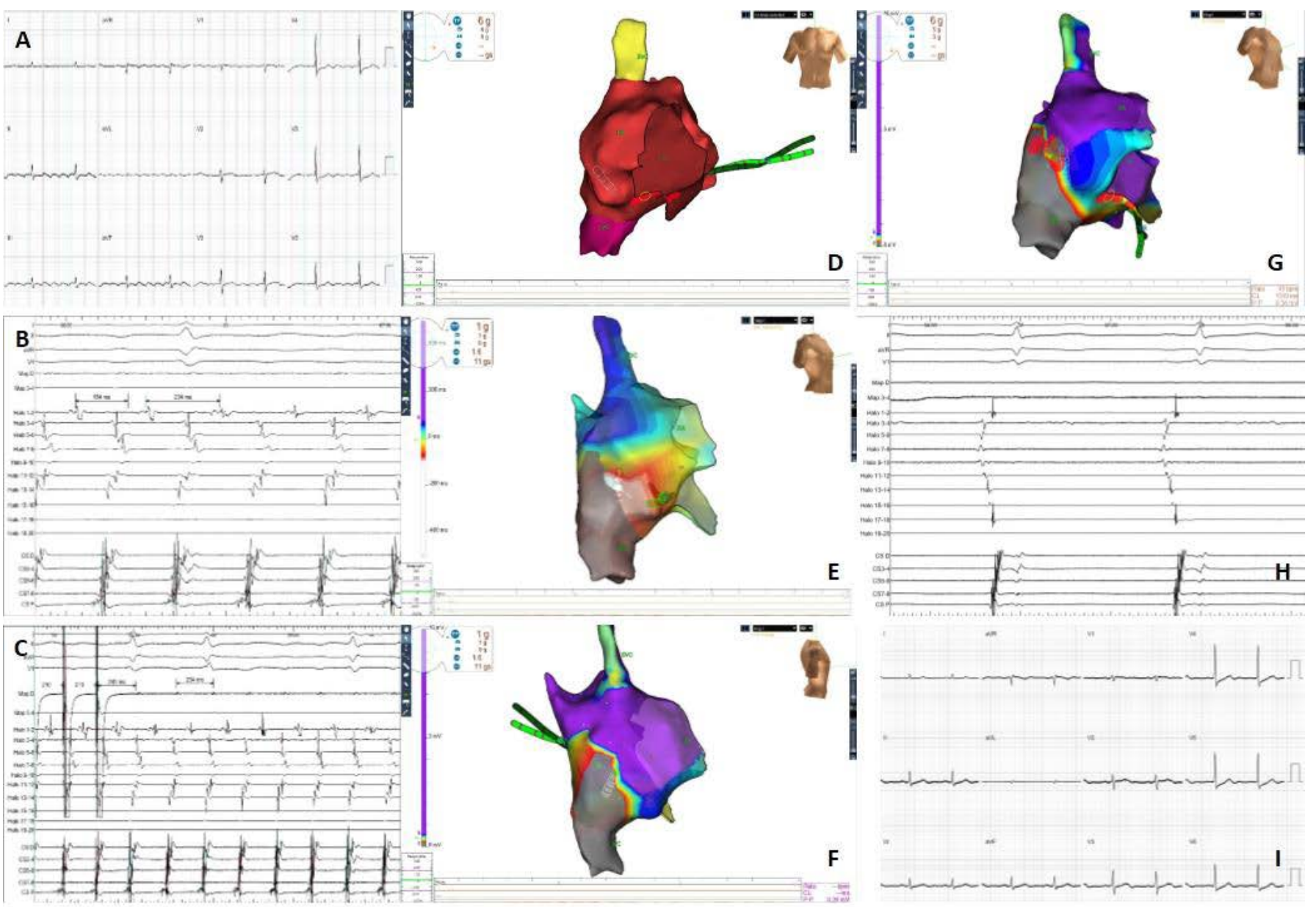

the entrainment mapping, using an irrigated contact force sensing ablation catheter combined with activation, and voltage three-dimensional electroanatomical mapping, led to an efficient and reliable method to treat non-cavotricuspid isthmus-dependent atrial flutter occurring after cardiac surgery, or any other type of atrial scar.

\section{REFERENCES}

1. Steven D, Rostock T, Lutomsky B, Willems S. Three-dimensional mapping of atypical right atrial flutter late after chest stabbing. Pacing Clin Electrophysiol. 2008;31(3):382-385.

2. Yang $Y$, Cheng J, Bochoeyer A, et al. Atypical right atrial flutter patterns. Circulation. 2001;103:3092-8.

3. De Ponti R, Marazzi R, Zoli L, et al. Electroanatomic mapping and ablation of macroreentrant atrial tachycardia: comparison between successfully and unsuccessfully treated cases. J Cardiovasc Electrophysiol. 2010;21:155-62. 